Clinical trial

\title{
Cortical M1 plasticity and metaplasticity in patients with multiple sclerosis
}

\author{
Viola Baione $^{\mathrm{a}}$, Daniele Belvisi ${ }^{\mathrm{b}}$, Antonio Cortese ${ }^{\mathrm{a}}$, Ilaria Cetta ${ }^{\mathrm{a}}$, Matteo Tartaglia ${ }^{\mathrm{a}}$, \\ Enrico Millefiorini ${ }^{\mathrm{a}}$, Alfredo Berardelli ${ }^{\mathrm{a}, \mathrm{b}}$, Antonella Conte $\mathrm{e}^{\mathrm{a}, \mathrm{b}, *}$ \\ a Department of Human Neurosciences, Sapienza, University of Rome, Viale dell' Università 30, 00185 Rome, Italy \\ ${ }^{\mathrm{b}}$ IRCCS Neuromed, via Atinense 18, 86077 Pozzilli (IS), Italy
}

\section{A R T I C L E I N F O}

\section{Keywords:}

Multiple sclerosis

Theta burst stimulation

Cortical plasticity

Cortical metaplasticity

Motor performance

\begin{abstract}
A B S T R A C T
Background: Previous studies on patients with Multiple Sclerosis (MS) have reported contrasting findings on cortical plasticity of the primary motor cortex and no study has yet evaluated the regulatory mechanisms of cortical plasticity (i.e., metaplasticity) in MS patients. The aim of the present study was to investigate primary motor cortex (M1) plasticity and metaplasticity in patients with MS.

Methods: Nineteen patients affected by Relapsing--Remitting MS (RR-MS) and 16 age- and sex-matched healthy controls underwent intermittent Theta Burst Stimulation (iTBS) to evaluate cortical plasticity and iTBS preceded by repetitive index finger movements to evaluate M1 metaplasticity.

Results: In healthy subjects MEP size significantly increased after iTBS whereas it significantly decreased when repetitive index finger movements preceded iTBS (metaplasticity) (factor PROTOCOL: $p<0.0001$; PROTOCOL $x$ TIME interaction: $p=0.001$ ). Conversely, in MS patients MEP size mildly increased, albeit not significantly in both conditions $(p>0.05)$. In MS patients, percentage changes in MEP size induced by plasticity and metaplasticity protocol were significantly associated to EDSS $(p=0.001)$ and kinematics of index finger movements $(p=0.01)$.

Conclusion: M1 plasticity and metaplasticity are both altered in MS patients. When TBS is used for therapeutic purposes, TBS protocols should be tailored according to the M1 plasticity functional reserve of each MS patient.
\end{abstract}

\section{Introduction}

Studies in humans have shown that when repetitive transcranial magnetic stimulation (rTMS) is delivered over the primary motor cortex (M1), it induces long-lasting changes in the amplitude of motor evoked potentials (MEP), reflecting mechanisms of cortical plasticity (Ziemann et al., 2008). In healthy subjects the rTMS-induced effects on M1 largely depend on regulatory mechanisms of cortical plasticity. Metaplasticity is one of the regulatory mechanisms ensuring that synaptic plasticity remains within physiological and dynamic ranges, allowing learning of new tasks (Abraham and Bear, 1996).

In patients with Multiple Sclerosis (MS) several studies have investigated cortical plasticity by means of various rTMS protocols but the results on cortical plasticity in MS are still controversial (Zeller et al., 2012; Mori et al., 2011, 2012, 2013, 2016; Conte et al., 2016; Wirshing et al., 2018). The authors who reported increased cortical plasticity or paradoxical excitatory after-effects induced by rTMS (Mori et al., 2013, 2014), suggested that the inflammatory excitotoxic damage (Mori et al., 2012, 2014) may be responsible for abnormal plasticity mechanisms in MS patients. Recent studies have also suggested that rTMS (Iodice et al., 2017) may be used as a therapeutic strategy to improve motor functions in these patients (Centonze et al., 2007; Mori et al., 2009, 2010; Boutière et al., 2017). It is possible that in patients with MS inflammatory damage not only affects cortical plasticity but also its regulatory mechanisms, namely cortical metaplasticity. Understanding whether regulatory mechanisms of cortical plasticity are altered in MS has important implications when rTMS protocols are to be applied for therapeutic purposes.

In order to determine whether cortical plasticity in MS is altered and, if so, whether the alteration is related to impaired regulatory mechanisms of plasticity, i.e., metaplasticity, we investigated both cortical plasticity and cortical metaplasticity mechanisms in a cohort of Relapsing-Remitting MS (RRMS) patients. The results were compared with those from a control group of healthy subjects.

\footnotetext{
* Corresponding author at: Department of Human Neurosciences, Sapienza, University of Rome, Viale dell' Università 30 , 00185 Rome, Italy.

E-mail address: antonella.conte@uniroma1.it (A. Conte).
} 
Table 1

Demographic and clinical features of patients with relapsing-remitting multiple sclerosis.

\begin{tabular}{|c|c|c|c|c|c|}
\hline & Age (years) & Gender & $\begin{array}{l}\text { Disease duration } \\
\text { (years) }\end{array}$ & EDSS & DMD \\
\hline 1 & 37 & $\mathrm{~F}$ & 9 & 4 & Teriflunomide \\
\hline 2 & 40 & M & 10 & 1 & Dimethylfumarate \\
\hline 3 & 32 & $\mathrm{~F}$ & 3 & 3 & Dimethylfumarate \\
\hline 4 & 24 & $\mathrm{~F}$ & 1 & 3,5 & Dimethylfumarate \\
\hline 5 & 51 & M & 14 & 2 & IFN $\beta-1$ a 44 mcg sc \\
\hline 6 & 48 & $\mathrm{~F}$ & 28 & 0 & Dimethylfumarate \\
\hline 7 & 32 & M & 17 & 2 & Dimethylfumarate \\
\hline 8 & 46 & $\mathrm{~F}$ & 23 & 2,5 & IFN $\beta-1$ a 44 mcg sc \\
\hline 9 & 30 & $\mathrm{~F}$ & 2 & 2 & Dimethylfumarate \\
\hline 10 & 57 & $\mathrm{~F}$ & 19 & 3 & IFN $\beta-1$ a 30 mcgim \\
\hline 11 & 37 & M & 10 & 1 & IFN $\beta-1$ a 44 mcg sc \\
\hline 12 & 33 & $\mathrm{~F}$ & 10 & 3 & Fingolimod \\
\hline 13 & 36 & $\mathrm{~F}$ & 1 & 1 & IFN $\beta-1 \mathrm{a} 44 \mathrm{mcg} s \mathrm{c}$ \\
\hline 14 & 34 & $\mathrm{~F}$ & 4 & 0 & Dimethylfumarate \\
\hline 15 & 35 & $\mathrm{~F}$ & 6 & 1 & IFN $\beta-1 \mathrm{a} 44 \mathrm{mcg} \mathrm{sc}$ \\
\hline 16 & 25 & $\mathrm{~F}$ & 3 & 1,5 & Fingolimod \\
\hline 17 & 45 & $\mathrm{~F}$ & 3 & 1 & Teriflunomide \\
\hline 18 & 45 & $\mathrm{~F}$ & 4 & 4 & Dimethylfumarate \\
\hline 19 & 42 & $\mathrm{~F}$ & 15 & 4 & Dimethylfumarate \\
\hline
\end{tabular}

Abbreviations: F: female; M: male; EDSS: Expanded disability Status Scale; DMD: disease modifying drug.

\section{Material and methods}

\subsection{Subjects and clinical assessment}

Nineteen RRMS patients were consecutively enrolled in the study and 16 age- and sex-matched healthy controls (HC) acted as control group. The participants' demographic and clinical characteristics are reported in Table 1 . The inclusion criteria were age over 18 years; diagnosis of MS according to the McDonald criteria (Polman et al., 2011); no disease relapse or corticosteroid intake in the last 30 days; MiniMental State Examination (MMSE) $>28$; Expanded Disability Status Scale (EDSS) $\leq 4$ (Kurtzke, 1983). All the patients enrolled were on Disease Modifying Drugs (DMDs). The study was approved by the Ethics Committee of Policlinico Umberto I, Sapienza University of Rome, and was conducted in accordance with the Declaration of Helsinki. Written informed consent was obtained from every participant before the beginning of the study.

\subsection{Neurophysiological assessment: stimulation techniques}

Subjects were studied in two experimental sessions separated by approximately one week. All the participants were comfortably seated in an armchair. Single TMS pulses were delivered by means of a monophasic Magstim stimulator and intermittent Theta Burst Stimulation (iTBS) was delivered by means of a biphasic Super Rapid Magstim 200 magnetic stimulator (Magstim, Whitland, Dyfed, UK). Both stimulators were connected to a figure eight coil (external wing $9 \mathrm{~cm}$ in diameter) placed tangentially over the scalp with the handle pointing back and away from the midline at $45^{\circ}$, which is the optimal position for eliciting MEP in the contralateral target muscle (first dorsal interosseous-FDI- in the dominant hand).

Motor thresholds, which were calculated in both sessions at rest (Resting Motor Threshold - RMT), were defined as the lowest stimulus intensity able to evoke a MEP of an amplitude of $\geq 50 \mu \mathrm{V}$ in 5 out of 10 consecutive trials, and during a slight voluntary contraction (Active Motor Threshold -AMT) of the target muscle (20\%-30\% of the maximum voluntary contraction), which was defined as the lowest intensity able to evoke a MEP of $\geq 200 \mathrm{~V}$ in 5 out of 10 consecutive trials. The intensity of the single-pulse TMS used to monitor changes in cortical excitability was set so as to obtain a mean MEP size of $1 \mathrm{mV}$ at the baseline. iTBS, which was applied according to a previously described technique (Huang et al., 2005; Iezzi et al., 2008, 2011), consisted of bursts given in short trains lasting $2 \mathrm{~s}$ (i.e., 10 bursts) repeated every $10 \mathrm{~s}$ for a total number of 600 pulses (i.e., 20 trains). iTBS was delivered at $80 \%$ of the AMT intensity.

\subsection{EMG recording}

Electromyographic (EMG) activity was recorded through a pair of $\mathrm{Ag} / \mathrm{AgCl}$ electrodes placed over the FDI muscle in a belly-tendon fashion. The raw EMG signal was amplified and filtered (bandwidth: $20 \mathrm{~Hz}-1 \mathrm{kHz}$ ) by means of a Digitimer D360 amplifier (Digitimer) and sampled at $5 \mathrm{kHz}$ (CED 1401 A/D laboratory interface, Cambridge Electronic Design, Cambridge, UK). Data were stored on a laboratory computer for on-line visual display and for further off-line analysis (Signal software, Cambridge Electronic Design). EMG activity of the target muscle was monitored throughout the experimental sessions by using an audio and high-gain visual feedback to ensure the complete relaxation of the target muscles. Baseline MEP size was measured peakto-peak. MEP size after movements and after TBS was expressed as a percentage of the baseline MEP.

\subsection{Motor task and movement recording}

Participants were comfortably seated in an armchair beside a table. The arm was abducted at the shoulder by about $45-50^{\circ}$, and the elbow joint was flexed at about $90^{\circ}$ After a verbal "go" signal, subjects abducted the index finger, then returned the finger to the starting position upon being given a verbal "stop" signal shortly after. Each subject performed 30 index finger abductions, preceded by 3 or 4 training movements. Subjects were instructed to perform movements "as widely and as quickly as possible" after the verbal command and were continually encouraged to do so throughout the motor task. Index finger movements in the three-dimensional space were recorded by means of the SMART analyzer motion system (BTS Engineering, Milan, Italy), which contains three infrared cameras (sampling rate, $120 \mathrm{~Hz}$ ) that follow the displacement of a passive marker taped on the distal phalanx of the subject's right index finger. A dedicated software was used to reconstruct the displacement of the passive marker off-line and automatically determine the kinematic features of each movement. The same software was used to measure Range Of Motion (ROM) and angular speed (Conte et al., 2017, 2018).

\subsection{Experimental paradigm}

The experimental procedures consisted in two sessions whose order of presentation was pseudorandomized across subjects.

\subsubsection{Cortical plasticity protocol}

In this session, we tested the effects of iTBS on the motor cortex plasticity by using single TMS pulses delivered at rest over the FDI motor cortical hot spot before (baseline, T0) and 5 (T1), 15 (T2) and $30 \mathrm{~min}$ (T3) after iTBS. We collected 10 MEPs at each time point.

\subsubsection{Metaplasticity protocol: effects of index finger movements on iTBS after-effects}

In this session, we investigated the effects of index finger movements on iTBS-induced after-effects. Single TMS pulses were delivered before (T0), immediately after index finger movements (MEP post-mov) and 5 (T1), 15 (T2) and $30 \mathrm{~min}$ (T3) after iTBS. We also evaluated motor performance during the task by using a kinematic analysis to measure the mean amplitude and mean peak velocity of index finger abductions.

\subsection{Statistical analysis}

We used the SPSS 25.0 toolbox (version 25, IBM, New York, USA) 
for all the statistics. Group comparisons were tested by means of the Shapiro-Wilks test to evaluate whether distribution was Gaussian or not, and parametric (Repeated measure ANOVA, One-way ANOVA) or non-parametric tests (Mann-Whitney U test) were used accordingly.

For the analysis of the neurophysiological data, we used a betweengroups ANOVA to compare the kinematic parameters of the index finger movements (ROM, mean angular velocity and time) and TMS variables (AMT, intensity to evoke $1 \mathrm{mV}$ MEP amplitude) between MS patients and healthy subjects. We then compared percentage changes in MEP amplitude induced by iTBS (plasticity protocol) and iTBS preceded by index finger movements (metaplasticity protocol) in MS patients and healthy subjects using a repeated measures ANOVA with factor GROUP (2 levels: MS vs. healthy) and factors PROTOCOL (2 levels: iTBS vs. iTBS preceded by index finger movements) and TIME (baseline, T1, T2, T3).

Correlations (Pearson's correlation coefficient) and a linear regression analysis were performed to investigate any association between the neurophysiological and clinical variables. Pearson's correlation coefficient was also performed to investigate any association between mean percentage changes in MEP amplitude induced by plasticity and metaplasticity interventions. All the results are reported at $p<0.05$ after FDR correction for multiple comparisons.

\section{Results}

\subsection{TMS variables at baseline and kinematic parameters in MS patients and} healthy subjects

One-way ANOVA showed a similar baseline MEP amplitude in MS patients and healthy subjects ( $p=0.77)$, though AMT and intensity for $1 \mathrm{mV}$ MEP were higher in MS patients than in healthy subjects $(p<0.05)$. One-way ANOVA also showed that the kinematic parameters (ROM, mean angular velocity and time) of index finger movements were similar in MS and healthy subjects (all ps > 0.05).

\subsection{Changes in MEP amplitude induced by the cortical plasticity and metaplasticity protocol in MS patients and healthy subjects}

Repeated measures ANOVA for MEP amplitude percentage changes revealed a significant factor PROTOCOL $(F=5.4, p=0.02)$, a significant GROUP x PROTOCOL interaction $(F=5.7, p=0.02)$ and a significant GROUP $\mathrm{x}$ PROTOCOL $\mathrm{x}$ TIME interaction $(F=2.8$, $p=0.04$ ). Post-hoc analysis showed that the MEP amplitude in healthy subjects increased after iTBS but decreased when index finger movement preceded iTBS (factor PROTOCOL: $F=24.6, p<0.0001$; PROTOCOL x TIME interaction: $F=6.6, p=0.001$ ) (Fig. 1).

By contrast, the MEP amplitude in MS patients increased in both sessions, albeit not significantly (all ps $>0.05$ ) (see Fig. 2).

Linear regression analysis showed that the changes in the MEP amplitude between the two sessions (mean percentage changes in MEP amplitude in the "metaplasticity" session/mean percentage changes in MEP amplitude in the "cortical plasticity" session) were significantly associated with the EDSS score ( $t=4.34, p=0.001$ ) (see Fig. 3 ), mean angular velocity $(t=-2.47, p=0.02)$ and duration of the index finger movements $(t=-2.9, p=0.01)$, though not with AMT or intensity for $1 \mathrm{mV}$ MEP.

No correlation was detected between MEP changes induced by the "cortical plasticity" and "cortical metaplasticity" protocols in MS patients $(p>0.05)$.

\subsection{Effect of the motor task on plasticity in the primary motor area}

Repeated measures ANOVA revealed a significant factor "motor TASK" $(F=5.6 ; p=0.02)$ though not a significant factor "Group" $(F=0.53, p=0.39)$ and MOTOR TASK $\mathrm{x}$ GROUP interaction $(F=1,7$; $p=0.2$ ), which indicates that the increase in MEP amplitude

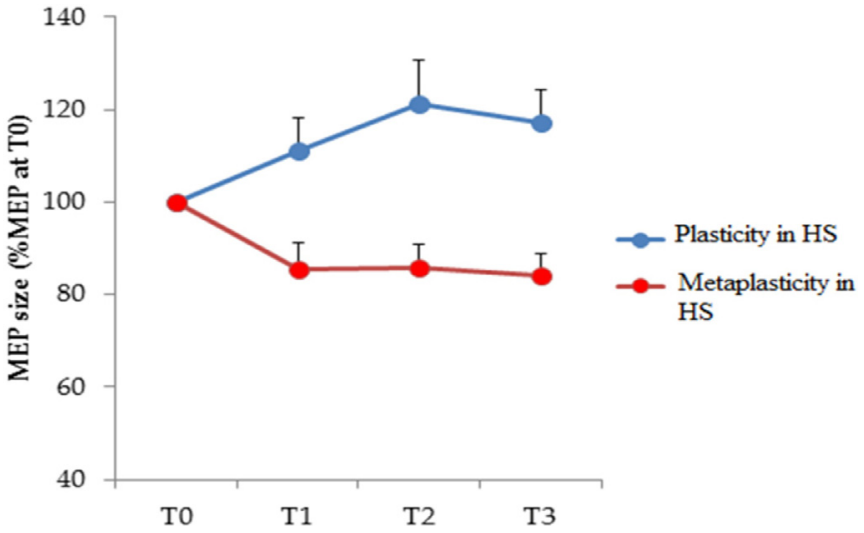

Fig. 1. Percentage changes in motor evoked potential (MEP) amplitude induced by intermittent Theta Burst Stimulation (iTBS) (plasticity protocol) and iTBS preceded by index finger movements (metaplasticity protocol) in healthy subjects.

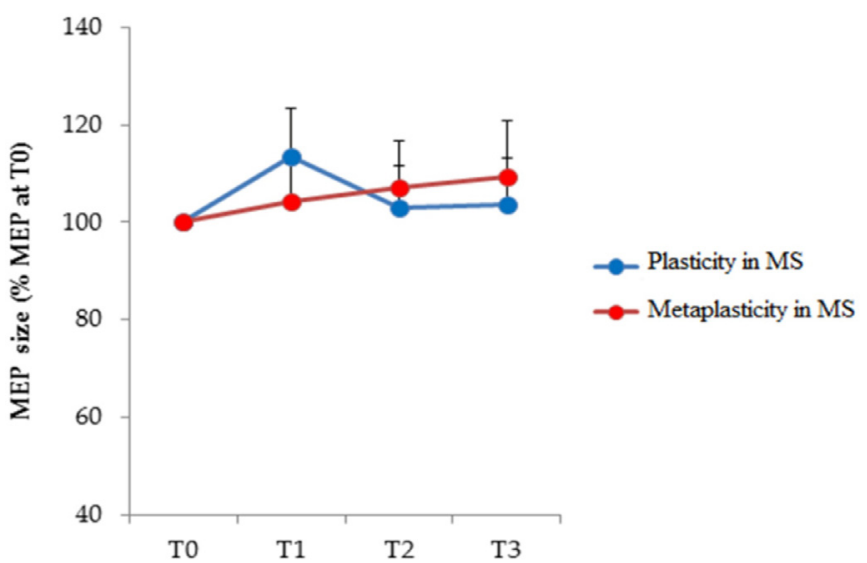

Fig. 2. Percentage changes in motor evoked potential (MEP) amplitude induced by intermittent Theta Burst Stimulation (iTBS) (plasticity protocol) and iTBS preceded by index finger movements (metaplasticity protocol) in patients with multiple sclerosis (MS).

immediately after the index finger movements was similar in MS patients and healthy controls.

\section{Discussion}

Two main findings emerge from our study. The first is that following iTBS over M1 the MEP amplitude increased to a lesser extent in MS patients (plasticity protocol) than in healthy subjects. The second finding is that when the repetition of voluntary index finger abductions preceded iTBS (metaplasticity protocol), the MEP amplitude decreased in healthy subjects (Iezzi et al., 2008) whereas in MS patients it increased slightly. Taken together, these results suggest that both plasticity and metaplasticity in M1 are altered in MS patients.

When the experimental procedure was designed, various precautions were taken to avoid methodological errors that might bias the interpretation of the neurophysiological results. All the MS patients were studied in a remitting phase to ensure that the neurophysiological measurements were performed with the patients in a similar clinical phase. Moreover, all the subjects were studied under the same experimental conditions and at the same time of day in order to reduce any variability caused by circadian rhythms. The experimental sessions took place at least one week apart to exclude take-over effects (Bienenstock et al., 1982; Siebner et al., 2009). The subjects were instructed to relax completely in order to avoid any muscle contractions that might alter the MEP size (Huang et al., 2008), and we continuously 


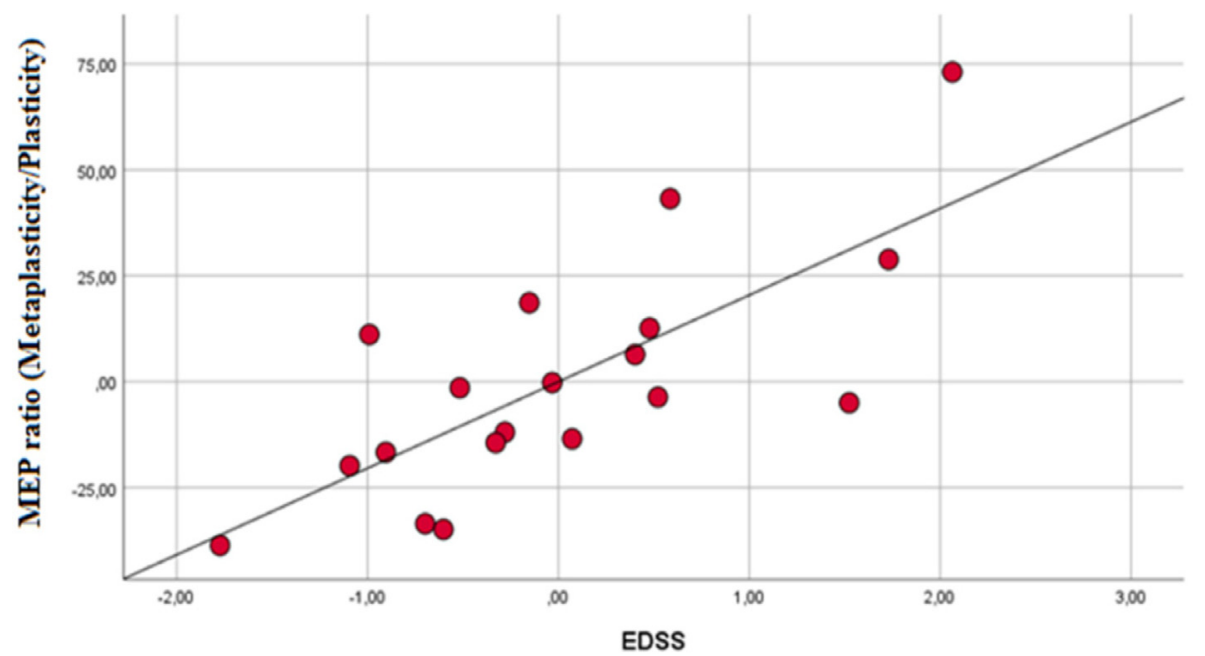

Fig. 3. Association between mean percentage changes in Motor Evoked Potential (MEP) amplitude between the two experimental sessions and Expanded Disability Status Scale (EDSS).

monitored the patients' EMG activity in the target muscles by means of visual and auditory feedback. During the motor task all the subjects were continually encouraged to perform the task "as widely and as quickly as possible" so as to ensure the correct execution and reliability of the subsequent analysis of the kinematic parameters of the movement itself. Although the stimulation intensity required to evoke both $1 \mathrm{mV}$ MEP and AMT was higher in MS patients than in healthy subjects, which points to reduced cortical excitability in M1 in the former, the stimulation performed at baseline elicited $1 \mathrm{mV}$ MEP in both the MS patients and healthy subjects. In addition, the intensities used in MS patients fell within a range corresponding to approximately half of the maximum power of the stimulator. We therefore believe that the altered cortical plasticity we detected following iTBS in MS does not depend on the stimulation intensity.

\subsection{Cortical plasticity in MS patients and in healthy subjects}

Previous studies have reported normal iTBS-induced cortical plasticity in stable RR-MS patients but a loss of plasticity during relapses in RR-MS patients and in primary progressive-MS (Mori et al., 2012, 2013). Other authors have instead reported normal plasticity (Zeller et al., 2010, 2012). Here we found that cortical plasticity is abnormal when RR-MS patients are studied in the remitting phase. The discrepancies in the findings between the various studies may be ascribed to several factors. Most previous studies performed on MS patients did not include a control group and the intensity of TMS stimulation was set so as to obtain a varying MEP amplitude range at baseline (0.5-1 mV) (Mori et al., 2013). In addition, patients were classified as "responders to rTMS protocol" when the ratio between the mean percentage changes in MEP size post-iTBS and the mean percentage changes in MEP size at baseline was higher than "1". By applying the same methodology, the MS patients we studied were normal in terms of cortical plasticity, whereas their response to iTBS was reduced if compared with that of healthy controls. A MEP ratio higher than " 1 " does not, therefore, necessarily imply normal iTBS-induced plasticity. The discrepancies in the cortical plasticity findings in MS patients between different studies may thus depend on methodological issues.

The reduced response to iTBS over M1 observed in our MS patients is likely to depend on altered ion-channel activation (Ziemann et al., 2008). As demonstrated in animal models of MS (Experimental Autoimmune Encephalomyelitis -EAE) (Stampanoni Bassi et al., 2017), proinflammatory cytokines lead to marked changes in synaptic functioning and to an ion channel dysfunction from the earliest stages of the disease (Arnold et al., 2015). Inflammation in MS subverts the physiological balance between excitatory and inhibitory transmission and results in enhanced glutamatergic activation, which seems to play a key role in disease progression and in the establishment of the neurodegenerative process (Centonze et al., 2010; Rossi et al., 2012).

One possible explanation for cortical plasticity alterations in MS is, as has previously been suggested (Rossi et al., 2012; Nisticò et al., 2013), the presence of a widespread inflammatory microenvironment that reduces GABAergic inhibitory transmission to the benefit of NMDA receptor-mediated excitatory glutamate transmission. This persistent glutamate NMDA receptor activation leads to neurotoxic processes that worsen neuronal dysfunction, as has been shown in numerous degenerative disorders including amyotrophic lateral sclerosis and Alzheimer's disease (Matute, 2011; Kostic et al., 2017). Moreover, glutamate excitotoxicity may lead to abnormalities in LTP-like cortical plasticity, as tested by means of rTMS techniques, that resemble those reported in Alzheimer's disease (Di Lazzaro et al., 2002; Nardone et al., 2014).

\subsection{Metaplasticity in MS patients and healthy subjects}

The metaplasticity protocol used in MS patients in our study yielded opposite effects to those observed in healthy subjects. Since the kinematic parameters (ROM, mean angular velocity and time) of the index finger movements preceding the iTBS cortical plasticity induction in MS patients were comparable to those in healthy controls, we rule out the possibility that the lack of metaplastic effect was due to poor motor performance. Moreover, the observation of a similar degree of postmovement MEP amplitude facilitation in healthy subjects and MS patients confirms that MS patients effectively performed index finger movements and motor practice. We may also assume that the postmovement facilitation mechanisms in the MS patients we studied, whose level of clinical impairment was shown to be low by the EDSS, were functionally intact.

In healthy subjects, the execution of short sequences of repeated movements reverses the polarity of cortical excitability through mechanisms that depend on the activation of voltage-controlled calcium channels and are termed "polarity-reversing metaplasticity" (Gentner et al., 2008; Iezzi et al., 2008). The physiological function of cortical metaplasticity, also defined as "plasticity of synaptic plasticity", is that of maintaining neuronal synaptic plasticity within a dynamic range of activity (Abraham and Bear, 1996), thus stabilizing neuronal activity. According to the Bienenstock-Cooper-Munro theory, the induction threshold of LTP and LTD is dynamically adapted according to the previous post-synaptic activity level through homeostatic mechanisms, thereby preventing excessive expression of LTP or LTD. Our 
observations now suggest that not only mechanisms of plasticity but also those of metaplasticity are altered in MS patients.

\subsection{Relationship between cortical plasticity and metaplasticity}

By testing both plasticity and metaplasticity protocols in the same patients, we observed that the extent of the MEP changes induced by the plasticity protocol was not linearly related to that induced by the metaplasticity protocol. Although plasticity and metaplasticity mechanisms share several molecular and cellular pathways (Yee et al., 2017; Li et al., 2018; Henderson et al., 2018), they may be affected by the pathophysiological mechanisms with different temporal and spatial ranges. Indeed, while metaplasticity mechanisms occur over a very slow time course and involve all the synapses of a neuron so as to ensure the stability and synchronize the firing rate of a neuronal population, synaptic plasticity may occur over a very short period and affect individual synapses (homosynaptic mechanism) (Yee et al., 2017; Henderson et al., 2018).

\subsection{Relationship between cortical plasticity and metaplasticity with motor performance}

The other noteworthy finding of the present study is that there is an association in MS patients between changes in MEP amplitude between the two sessions (iTBS and iTBS preceded by movement) and both the EDSS scores and the kinematic movement parameters (angular velocity and duration of movement). This association may reflect abnormal nonhomeostatic cortical plasticity induced by motor learning (Halsband and Lange, 2006). Although the kinematic properties MS patients use to perform index finger abductions are similar to those adopted by healthy subjects, a better execution of the motor task preceding the delivery of the iTBS paradigm in individual patients may result in a greater degree of metaplasticity. This finding therefore suggests that the extent of metaplasticity is associated not only with the clinical severity of the disease as expressed by the EDSS, but also with motor performance and the kinematic parameters of the motor task.

\section{Conclusion}

In conclusion, the present study shows that M1 plasticity and metaplasticity mechanisms are altered in patients with MS. To place our findings within a clinical perspective, the application of rTMS protocols in MS to "boost" rehabilitative strategies should be tailored to each patient since the effects of the rTMS protocol in MS do not necessarily yield the polarity and the same effects as those observed in healthy subjects.

\section{Declaration of Competing Interest}

No competing interests to declare.

\section{Funding}

This research did not receive any specific grant from funding agencies in the public, commercial, or not-for-profit sectors.

\section{References}

Abraham, W.C., Bear, M.F., 1996. Metaplasticity: the plasticity of synaptic plasticity. Trends Neurosci. 19, 126-130.

Arnold, R., Huynh, W., Kiernan, M.C., Krishnan, A.V., 2015. Ion channel modulation as a therapeutic approach in multiple sclerosis. Curr. Med. Chem. 22, 4366-4378.

Bienenstock, E.L., Cooper, L.N., Munro, P.W., 1982. Theory for the development of neuron selectivity: orientation specificity and binocular interaction in visual cortex. J. Neurosci. 2, $32-48$.
Boutière, C., Rey, C., Zaaraoui, W., et al., 2017. Improvement of spasticity following intermittent theta burst stimulation in multiple sclerosis is associated with modulation of resting-state functional connectivity of the primary motor cortices. Mult. Scler. 23, $855-863$.

Centonze, D., Koch, G., Versace, V., et al., 2007. Repetitive transcranial magnetic stimulation of the motor cortex ameliorates spasticity in multiple sclerosis. Neurology 68, 1045-1050.

Centonze, D., Muzio, L., Rossi, S., Furlan, R., Bernardi, G., Martino, G, 2010. The link between inflammation, synaptic transmission and neurodegeneration in multiple sclerosis. Cell Death Differ. 17, 1083-1091.

Conte, A., Li Voti, P., Pontecorvo, S., et al., 2016. Attention-related changes in short-term cortical plasticity help to explain fatigue in multiple sclerosis. Mult. Scler. 22, 1359-1366.

Conte, A., Belvisi, D., Tartaglia, M., et al., 2017. Abnormal temporal coupling of tactile perception and motor action in Parkinson's disease. Front. Neurol. 8, 249.

Conte, A., Belvisi, D., De Bartolo, M.I., et al., 2018. Abnormal sensory gating in patients with different types of focal dystonias. Mov. Disord. 33, 1910-1917.

Di Lazzaro, V., Oliviero, A., Tonali, P.A., et al., 2002. Noninvasive in vivo assessment of cholinergic cortical circuits in AD using transcranial magnetic stimulation. Neurology 59 , 392-397.

Gentner, R., Wankerl, K., Reinsberger, C., Zeller, D., Classen, J., 2008. Depression of human corticospinal excitability induced by magnetic theta-burst stimulation: evidence of rapid polarity-reversing metaplasticity. Cereb. Cortex 18, 2046-2053.

Halsband, U., Lange, R.K., 2006. Motor learning in man: a review of functional and clinical studies. J. Physiol. Paris 99, 414-424.

Henderson, J.A., Gong, P., 2018. Functional mechanisms underlie the emergence of a diverse range of plasticity phenomena. PLoS Comput. Biol. 14, e1006590.

Huang, Y.Z., Edwards, M.J., Rounis, E., Bhatia, K.P., Rothwell, J.C., 2005. Theta burst stimulation of the human motor cortex. Neuron 45, 201-206.

Huang, Y.Z., Rothwell, J.C., Edwards, M.J., Chen, R.S., 2008. Effect of physiological activity on an NMDA-dependent form of cortical plasticity in human. Cereb. Cortex 18, 563-570.

Iezzi, E., Conte, A., Suppa, A., et al., 2008. Phasic voluntary movements reverse the aftereffects of subsequent theta-burst stimulation in humans. J. Neurophysiol. 100, 2070-2076.

Iezzi, E., Suppa, A., Conte, A., Li Voti, P., Bologna, M., Berardelli, A., 2011. Short-term and longterm plasticity interaction in human primary motor cortex. Eur. J. Neurosci. 33, 1908-1915.

Iodice, R., Manganelli, F., Dubbioso, R., 2017. The therapeutic use of non-invasive brain stimulation in multiple sclerosis - a review. Restor. Neurol. Neurosci. 35, 497-509.

Kostic, M., Zivkovic, N., Cvetanovic, A., Stojanovic, I., Colic, M., 2017. IL-17 signalling in astrocytes promotes glutamate excitotoxicity: indications for the link between inflammatory and neurodegenerative events in multiple sclerosis. Mult. Scler. Relat. Disord. 11, 12-17.

Kurtzke, J.F., 1983. Rating neurologic impairment in multiple sclerosis: an expanded disability status 19 scale (EDSS). Neurology 33, 1444-1452.

Li, J., Park, E., Zhong, L.R., Chen, L., 2018. Homeostatic synaptic plasticity as a metaplasticity mechanism - a molecular and cellular perspective. Curr. Opin. Neurobiol. 54, 44-53.

Matute, C., 2011. Glutamate and ATP signalling in white matter pathology. J. Anat. 219, 53-64.

Mori, F., Codecà, C., Kusayanagi, H., et al., 2010. Effects of intermittent theta burst stimulation on spasticity in patients with multiple sclerosis. Eur. J. Neurol. 17, 295-300.

Mori, F., Koch, G., Foti, C., Bernardi, G., Centonze, D., 2009. The use of repetitive transcranial magnetic stimulation (rTMS) for the treatment of spasticity. Prog. Brain Res. 175, 429-439.

Mori, F., Kusayanagi, H., Buttari, F., et al., 2012. Early treatment with high-dose interferon beta-1a reverses cognitive and cortical plasticity deficits in multiple sclerosis. Funct. Neurol. 27, 163-168.

Mori, F., Kusayanagi, H., Nicoletti, C.G., et al., 2014. Cortical plasticity predicts recovery from relapse in multiple sclerosis. Mult. Scler. 20, 451-457.

Mori, F., Nisticò, R., Nicoletti, C.G., et al., 2016. RANTES correlates with inflammatory activity and synaptic excitability in multiple sclerosis. Mult. Scler. 22, 1405-1412.

Mori, F., Rossi, S., Piccinin, S., et al., 2013. Synaptic plasticity and PDGF signaling defects underlie clinical progression in multiple sclerosis. J. Neurosci. 33, 19112-19119.

Mori, F., Rossi, S., Sancesario, G., et al., 2011. Cognitive and cortical plasticity deficits correlate with altered amyloid- $\beta$ CSF levels in multiple sclerosis. Neuropsychopharmacology 36, 559-568.

Nardone, R., Tezzon, F., Höller, Y., Golaszewski, S., Trinka, E., Brigo, F, 2014. Transcranial magnetic stimulation (TMS)/repetitive TMS in mild cognitive impairment and Alzheimer's disease. Acta Neurol. Scand. 129, 351-366.

Nisticò, R., Mango, D., Mandolesi, G., et al., 2013. Inflammation subverts hippocampal synaptic plasticity in experimental multiple sclerosis. PLoS One 8, e54666.

Polman, C.H., Reingold, S.C., Banwell, B., et al., 2011. Diagnostic criteria for multiple sclerosis: 2010 revisions to the McDonald criteria. Ann. Neurol. 69, 292-302.

Rossi, S., Furlan, R., De Chiara, V., et al., 2012. Interleukin-1 $\beta$ causes synaptic hyperexcitability in multiple sclerosis. Ann. Neurol. 71, 76-83.

Siebner, H.R., Hartwigsen, G., Kassuba, T., Rothwell, J.C., 2009. How does transcranial magnetic stimulation modify neuronal activity in the brain? Implications for studies of cognition. Cortex 45, 1035-1042.

Stampanoni Bassi, M., Mori, F., Buttari, F., Marfia, G.A., Sancesario, A., Centonze, D., Iezzi, E., 2017. Neurophysiology of synaptic functioning in multiple sclerosis. Clin. Neurophysiol. 128, 1148-1157.

Wirsching, I., Buttmann, M., Odorfer, T., Volkmann, J., Classen, J., Zeller, D., 2018. Altered motor plasticity in an acute relapse of multiple sclerosis. Eur. J. Neurosci. 47, 251-257.

Yee, A.X., Hsu, Y.T., Chen, L., 2017. A metaplasticity view of the interaction between homeostatic and Hebbian plasticity. Philos. Trans. R. Soc. Lond. B. Biol. Sci. 372 (1715). https://doi.org/10.1098/rstb.2016.0155.

Zeller, D., aufm Kampe, K., Biller, A., et al., 2010. Rapid-onset central motor plasticity in multiple sclerosis. Neurology 74, 728-735.

Zeller, D., Dang, S.Y., Weise, D., Rieckmann, P., Toyka, K.V., Classen, J., 2012. Excitability decreasing central motor plasticity is retained in multiple sclerosis patients. BMC Neurol. $12,92$.

Ziemann, U., Paulus, W., Nitsche, M.A., et al., 2008. Consensus: motor cortex plasticity protocols. Brain Stimul. 1 (3), 151-163. 